\title{
La axiología educativa para mejorar la inteligencia emocional y su importancia en la formación de docentes
}

\section{Educational axiology to improve emotional intelligence and its importance in teacher training}

1 José Asencio Fonseca Carrasco

https://orcid.org/0000-0003-2979-2577.

Universidad Técnica de Ambato, Carrera de Educación Básica, Tungurahua,

ja.fonseca@uta.edu.ec

2 Carlos Alfredo Hernández Dávila (iD) https://orcid.org/0000-0002-2526-5051.

Universidad Técnica de Ambato, Carrera de Educación Básica, Tungurahua,

ca.hernandez@uta.edu.ec

Artículo de Investigación Científica y Tecnológica

Enviado: 24/12/2021

Revisado: 29/12/2021

Aceptado: $12 / 01 / 2022$

Publicado:08/03/2023

DOI: https://doi.org/10.33262/concienciadigital.v6i1.4.2032

Cótese: Fonseca Carrasco, J. A., \& Hernández Dávila, C. A. (2023). La axiología educativa para mejorar la inteligencia emocional y su importancia en la formación de docentes. ConcienciaDigital, $\quad 6(1.4)$, https://doi.org/10.33262/concienciadigital.v6i1.4.2032 843-861.

\begin{tabular}{l}
\hline CONCIENCIA DIGITAL, es una Revista Multidisciplinar, Trimestral, que se publicará en soporte electrónico \\
tiene como misión contribuir a la formación de profesionales competentes con visión humanística y crítica que \\
sean capaces de exponer sus resultados investigativos y científicos en la misma medida que se promueva \\
mediante su intervención cambios positivos en la sociedad. https://concienciadigital.org - \\
La revista es editada por la Editorial Ciencia Digital (Editorial de prestigio registrada en la Cámara Ecuatoriana \\
de Libro con No de Afiliación 663 ) www.celibro.org.ec \\
Esta revista está protegida bajo una licencia Creative Commons Attribution Non Commercial No Derivatives \\
4.0 International. Copia de la licencia: $\underline{\text { http://creativecommons.org/licenses/by-nc-nd/4.0/ }}$
\end{tabular}


Palabras claves:

axiología, valores, inteligencia emocional, currículo oculto, formación docente

Keywords: axiology, values, emotional intelligence, hidden curricula,

\section{Resumen}

Introducción. La axiología educativa posee una correlación intrínseca al valor, convirtiéndose en un fundamento y esencia del proceso de enseñanza, pues no existe otra alternativa de educar más que en valores. En la formación de docentes es imperioso mejorar la inteligencia emocional que permite controlar los sentimientos y las emociones del ser humano, de diferenciar entre ellos los que mejoran nuestra conducta y los que afecta a nuestro comportamiento y utilizar esa información para orientar nuestras acciones. Objetivo. Examinar la axiología educativa para mejorar la inteligencia emocional y su importancia en la formación de docentes de la carrera de educación básica en la Universidad Técnica de Ambato. Metodología. Se empleó un enfoque cuantitativo, el tipo de investigación aplicada fue de carácter explicativo analítico, la cual permitió ampliar el fenómeno de la inteligencia emocional basándose en tres aspectos tales como: atención, claridad y reparación, se usó el test basado en el Trait Meta-Mood Scale TMMS-24, el que consta de 24 preguntas con cinco alternativas que comprenden valores de 1 a 5 . Resultados. Basándonos en la encuesta demuestran tres aspectos: Atención: mujeres 69.6\%; hombres 55,6\% (adecuada atención). Claridad: mujeres 57,3\%; hombres $66,7 \%$ (adecuada comprensión). Reparación: mujeres 49.4\%; hombres 49.4,6\% (adecuada regulación). La población para esta investigación es de 30 estudiantes de primer nivel, 40 estudiantes de segundo nivel, 20 estudiantes de séptimo nivel y 20 estudiantes de octavo nivel. Conclusión. Se evidencia que los estudiantes poseen un adecuado nivel de inteligencia emocional con porcentajes similares en las tres dimensiones analizadas, también se identifica puntuaciones altas en la reparación emocional, en donde se destaca que, aunque a veces me siento triste suelo tener una visión optimista al igual que tengo mucha energía cuando me siento feliz coinciden en el porcentaje.

Abstract

Introduction. Educational axiology has an intrinsic correlation to value, becoming a foundation and essence of the teaching process, since there is no other alternative to educate but in values. In teacher training it is imperative to improve emotional intelligence, which allows us to control the feelings and emotions of human beings, to differentiate between those that improve our behavior and those that 
teacher education affect our behavior, and to use this information to guide our actions. Objective. To examine the educational axiology to improve emotional intelligence and its importance in the training of teachers of basic education at the Technical University of Ambato. Methodology. A quantitative approach was used, the type of research applied was of an explanatory-analytical nature, which allowed to expand the phenomenon of emotional intelligence based on three aspects such as: attention, clarity and repair, the test based on the Trait Meta-Mood Scale TMMS-24, which consists of 24 questions with five alternatives comprising values from 1 to 5 , was used. Results. Based on the survey, three aspects are demonstrated: Attention: women 69.6\%; men 55.6\% (adequate attention). Clarity: women 57.3\%; men $66.7 \%$ (adequate understanding). Repair: women $49.4 \%$; men $49.4 .6 \%$ (adequate regulation). The population for this research is 30 first level students, 40 second level students, 20 seventh level students and 20 eighth level students. Conclusion. It is evident that the students have an adequate level of emotional intelligence with similar percentages in the three dimensions analyzed, also high scores are identified in emotional repair, where it is highlighted that, although sometimes I feel sad, I usually have an optimistic vision as well as I have a lot of energy when I feel happy coincide in the percentage.

\section{Introducción}

La axiología educativa aparece relacionada con la Filosofía de la educación, la misma que esbozaba a los valores como prototipos que dirigen la conducta de los seres humanos hacia una actuación particular y a la evolución de la sociedad. La Pedagogía plantea la manera de llevarlos a la praxis, es decir educar en valores, tomando como referente las manifestaciones sociales y culturales de las comunidades humanas. La educación debería relacionarse permanente con la investigación axiológica y exclusivamente con los valores humanos y morales de manera que se pueda establecer una disposición con elementos consistentes e imprescindibles en el proceso de enseñanza aprendizaje, permitiendo establecer una respuesta a la diversidad de las realizaciones socioculturales del ser humano (Marin, 1987).

Un aspecto importante de la axiología como parte de la educación son los contenidos impartidos, pero relacionándolos con los valores mediante un proceso de formación del ser humano, la selección de estos debería responder al contexto actual en que se 
desenvuelve el hombre con el propósito de que le permita enfrentar problemáticas que tendrá que solucionar. Otro factor se constituye la destreza aplicada por los docentes y estudiantes en un aprendizaje consiente de la axiología a través de acciones formativas y auto formativas que se vinculan en el accionar y consolidación de la modelación del ser humano, premisa del educador. El trabajo intelectual y los valores se relacionan en el proceso educativo a través de una construcción humana a partir de la interpretación de la esencia de las cosas (Piotrowski, 1996).

La educación actual atraviesa una variedad de cambios vinculados con un periodo global de perdida de identidades nacionales, una economía que se basa en la producción de servicios, ante lo cual la institución educativa debe enfrentar realidades distintas, y adoptar el protagonismo de formar estudiantes que aprendan a convivir y de ser, con aptitudes para la vida y el desarrollo de su progreso. La idea de educar no se basa solo en saber, hacer y conocer, el estudiante debe aprender a ser y a convivir desde los establecimientos educativos, para ello las aptitudes sociales y emocionales son indispensables para coexistir en ambientes de desigualdad social. En la educación las experiencias sociales y emocionales permitirán solucionar problemáticas y trabajar de manera grupal para adoptar medidas de autoconciencia, autogestión y autocontrol que permitan regular las emociones propias. A través de la educación debe direccionarse la formación de entes solidarios e íntegros que permitirán edificar una sociedad diferente. Es necesario concebir ciudadanos para el siglo XXI que desarrollen competencias sociales y emocionales, para ello es necesario que la educación trabaje en un proyecto de valores (Hernández, 2005).

\section{Los valores}

Los seres humanos tienen la capacidad de discernir y formular reflexiones sobre su realidad circundante y al mismo tiempo expresar juicios de valor sobre las cosas. Los valores se mezclan con las cosas que en realidad son su esencia. Descifrar porque un objeto es bueno o malo es una capacidad intelectual del hombre, distinguir los valores es comprender que todo lo existente en el mundo es por algo y para algo, todo ser tiene su sentido y su razón de ser, todo cuanto existe es bueno y se constituye en un bien. De este modo podemos distinguir como valor aquello que hace buenas a las cosas, a las que consideramos como referentes de admiración y cumplimiento.

Según el congreso de la UNESCO realizado a finales del siglo anterior "Declaración Mundial sobre la Educación Superior en el siglo XXI", se examina que la sociedad actual vive una grave crisis de valores y se ha considerado que la educación superior tiene la finalidad de desarrollar y propender a la construcción de valores que promuevan una cultura de paz en toda la comunidad internacional, es inminente resguardar y fortalecer los valores de la sociedad, e infundir en los jóvenes un enfoque humanista que 
contribuyan a establecer una actuación ética.

Las sociedades a lo largo de la historia han delegado a la escuela como la encargada de trasmitir y desarrollar los valores a través de la actividad educativa, por lo tanto, la educación se ha convertido en el eslabón cultural que organiza, transfiere conocimientos, habilidades y actitudes que son aplicados por los individuos en un entorno comunitario. De esta manera la enseñanza ha aportado con la socialización de los valores a las jóvenes generaciones con el objetivo de avalar el respeto en la vida social y su prolongación. Es importante mencionar que la trasmisión de los valores en sociedades tradicionales ha sido elemental, más aún lo constituye en los actuales sistemas democráticos complejos y plurales, en donde la educación en valores permitirá mantener una convivencia pacífica, armónica y de cohesión social.

La familia se constituye en el ambiente propicio para la adquisición de los valores, sin embargo, no podemos afirmar que es la única agencia educativa socializadora en una colectividad, por esta aseveración es imposible establecer una separación entre familia y sociedad. El contexto familiar se contrapone a la actual sociedad con sus profundos cambios que inciden de un modo diverso a los padres y a los hijos. La sociedad occidental ha evidenciado varias transformaciones como en la defensa y ejercicio de las libertades, que se traducen en generalización de la educación, establecimiento de una cultura de tolerancia y una inserción del ciudadano en los asuntos públicos de la sociedad.

La educación en valores permite fortalecer la formación integral de los ciudadanos porque las actitudes se aprenden de manera diferente en lo que se refiere a los conocimientos y habilidades. Las conductas, el carácter se constituyen en componentes de la personalidad

de un individuo y que no solo recae sobre el educador sino también en el educando quien está influenciado por la cultura actual y al mismo tiempo debe tener una disposición al cambio.

Se determinan tres condiciones básicas para la educación en valores: la primera hace referencia a la motivación, intereses, valores, proyecto de vida, la segunda explorar el entorno circunstancial y la tercera identificar un modelo educativo ideal. El papel que desempeña el docente es trascendental su comportamiento ético y moral deben constituirse en estilos y formas de vida para que se imparta una educación en valores, la relación entre maestro y estudiante es concluyente, ya que se educa con el ejemplo de una convivencia diaria en la escuela.

\section{Inteligencia emocional}

Para definir el concepto de inteligencia emocional se toma como referencia a Salovey y Mayer y determinan que es "la capacidad para controlar los sentimientos y las emociones del ser humano, de diferenciar entre ellos los que mejoran nuestra conducta y los que 
afecta a nuestro comportamiento y utilizar esa información para orientar nuestras acciones“"(Salovey, 1990). Cabe indicar que esta definición fue posteriormente redefinida en vista de que se había prescindido la relación entre los sentimientos y el pensamiento, ante lo cual se menciona que "la inteligencia emocional es la habilidad para distinguir, evaluar, trasmitir y regular emociones, que promuevan el crecimiento emocional e intelectual" (Mayer, 1997).

- Se debe destacar el aporte de los autores antes mencionadas al haber investigado varias habilidades que se constituyen en compendios integrales de la competencia emocional.

- Examinar las emociones propias, cada persona puede identificar como se siente y a su vez direccionar y manejar sus emociones de manera reflexiva.

- Se debe controlar las emociones propias, cada persona tiene autonomía para esgrimir las renuencias emocionales, reemplazándolas por aptitudes conscientes que permitan la convivencia social.

- Desarrollar la capacidad personal, el mejoramiento escolar y social depende de varias condiciones como la persistencia, estimulación, la resiliencia, es decir tener seguridad en uno mismo.

- Tener empatía, debemos aprender a comunicarnos, saber escuchar, entender y respetar las ideas de las otras personas

- Establecer relaciones interpersonales, una relación satisfactoria debe estar enfocada en la resolución de conflictos personales, de cambiar las emociones negativas delos entes sociales (Mayer, 1997).

Se hace indispensable un cambio de paradigma en la formación de los futuros docentes que modelaran un nuevo estudiante que la sociedad futura necesita, adjudicando valores esenciales donde los ciudadanos proyecten su propio destino, pero buscando el bienestar de los demás entes de una comunidad. La Axiología educativa se constituye en el pilar de la coexistencia sosegada de un conglomerado humano basado en tres aspectos fundamentales, la educación en valores de colectivos autónomos, la educación en valores y la interculturalidad, la educación en valores para contrarrestar a la intimidación (Genis, 2005).

Se debe promover la convivencia pacífica a través de la cooperación entre las personas, cualidad innata que debe propiciar una interacción ciudadana en una sociedad civil. El objetivo del proceso educativo debe enfocarse en mejorar las competencias sociales y emocionales de sus estudiantes, indispensables para su desenvolvimiento en la vida pública, en sus labores diarias, y su preparación profesional (Exposito, 2018).

Según Delors (1997) en su obra “La educación encierra un tesoro“ se enfoca en relacionar la comunicación y el progreso personal y social. La educación del siglo XXI exigirá 
conocimientos teóricos y técnicos progresivos que se constituyen en las plataformas de las capacidades intelectuales del futuro. Con estas habilidades deberá filtrar la información transitoria que se encuentran en las áreas públicas y particulares y enfocarse en desarrollar proyectos de desarrollo propios y sociales. Para el autor es primordial el aprendizaje continuo como un ente integrador de reformas educativas y políticas pedagógicas. La educación deberá fundamentarse en cuatro pilares: aprender a conocer, aprender a hacer, aprender a vivir juntos, aprender a ser.

\section{La formación docente}

El conjugar la teoría con la praxis es trabajo primordial dentro de las actividades que desarrolla un docente, por lo que es fundamental que en la formación de los profesionales en el área educativa se tome en cuenta esta competencia para articular la labor del profesor juntamente con sus actitudes y aptitudes necesarias para avanzar en los retos que hoy en día se nos presentan. Además, es importante reconocer que el maestro es un ser humano que cuenta con un conjunto de creencias que hace de su accionar una labor única, por tal motivo es necesario una formación integral (Korthagen et al., 2006).

De acuerdo con Dewey (1938) manifiesta que la experiencia es un factor clave en una diversidad de circunstancias tomando en cuenta que pueden darse de diferentes formas, ya sean estas racionales, emotivas, conscientes e inconscientes. Por lo que la mayoría de las veces se recurre al ámbito del conocimiento y la conducta del ser humano que a su vez están dirigidos por los esquemas del pensamiento que dirigen el accionar de un individuo. Debido a esto la labor del docente en el proceso de construcción de un significado necesariamente debe hacer un llamado a la experiencia para que de esta manera exista un punto de partida hacia el desarrollo del aprendizaje. Llegamos al punto en donde la formación del profesorado es crucial, ya que necesariamente debe propiciar un ambiente idóneo en el que los estudiantes tengan las facilidades y el maestro sea un guía en el proceso de interpretación de cada alumno, porque para el desarrollo del conocimiento, no solo interviene el aspecto científico, sino que están inmersos de manera implícita las emociones, aptitudes, sentimientos, habilidades y valores de cada estudiante.

Contreras (2010) plantea que el objetivo de la educación no es la acumulación de contenidos descontextualizados, ya que esto no aporta a la generación de aprendizajes sólidos que colaboren a los estudiantes en la formación de ciudadanos útiles para la sociedad donde el conocimiento que disponen les ayude a resolver problemas de la vida cotidiana. Es así como se da paso a la creación de competencias, puesto que la persona conoce la situación a la que se enfrenta y sabe como debe actuar con los recursos que dispone en ese momento. Motivo por el cual la formación del docente no debe centrarse en la revisión de aspectos teóricos - científicos, más bien es necesario que el profesor con su ingenio plantee actividades con las que los alumnos se sientan motivados a reconstruir 
los conocimientos, habilidades y actitudes, porque de este modo el aprendizaje será más vivencial y comprenderá la verdadera relación entre la experiencia y el saber que se plasman en los contenidos disciplinares, ya que se ha puesto en un contexto conocido para el educando.

La diversidad que el estudiantado puede tener en un aula de clases es cada vez mayormente notario, debido a que no solo se trata de estatus social o nivel cultural, también el acceso a la información es un punto de quiebre por las facilidades que se brindan hoy en día por lo que es cada vez más complicado enganchar la atención del alumno, motivo por el cual es necesario una reformulación en la formación del profesional de educación para que cuente con las herramientas adecuadas y los recursos eficaces para llegar a los aprendices (Imbernón, 2001). De esta manera la idea cuando se habla de formación en el profesorado se tiene una mirada bipartita donde, por un lado, se trata de formar profesionales competentes que a su vez sean capaces guiar a sus dicentes en su andamiaje integral, es así, como la tarea del profesor debe ceñirse a los problemas que se presentan en el aula para que posteriormente cuente con las estrategias oportunas para enfrentar esta situación dejando de lado la enseñanza estándar para cambiar este paradigma por un esquema diversificado centrado en el aprendizaje continuo.

Otra de las problemáticas en el accionar docente es la repetición de la práctica pedagógica con un especial énfasis en la metodología, a pesar de la innovación en cuestión de recursos, herramientas tecnológicas, técnicas o instrumentos. De esta forma lo ratifica Tardif (2013) además considera que la formación del profesorado está clasificada en diferentes materias y teorías que deberían alinearse al conocimiento profesional, también están incluidos los esquemas mentales, objetivos, competencias, saberes y creencias. Todos estos aspectos no se pueden englobar en un libro, por lo que es importante integrar los conocimientos en las prácticas educativas que se encuentran reguladas por sus juicios, normativas y políticas.

\section{Currículo oculto}

Desde la posición de Santomé (1995) da a conocer que cuando nos referimos a currículo oculto se enfatiza en los aspectos relacionados con los valores y las normas que se encuentran incluidos de manera implícita en los planes de estudio de las instituciones educativas de la mano de los docentes quienes son los encargados de conjugar con sus estrategias y objetivos para encaminar la formación del alumnado. Además, indica que la escuela es un ente transmisor de hábitos, rutinas y disciplina, debido a que la organización de sus asignaturas son mediante horarios que también ayudan a que exista un respeto por el tiempo y el orden que se pretende integrar en los quehaceres de los estudiantes con el aporte de un comportamiento adecuado faciliten que los educandos vayan inculcando en sus vidas las normativas que posteriormente en el ámbito laboral se exige y 
complementado con un sistema de evaluación se muestra las debilidades y las fortalezas para potenciar su desarrollo.

Otra de las acepciones que se encuentran al tratar el currículo oculto son los textos que también poseen un trasfondo donde se pretende llegar con un conjunto de mensajes implícitos donde el conocimiento científico que se pretendía en un inicio dar a conocer se ve envuelto en un sinnúmero de prejuicios, sesgos o estereotipos que apartan del verdadero objetivo de la educación con un enfoque neutro, sino que más bien llevan a estudiante a inclinarse por cierta corriente ideológica (Torres, 1998).

La motivación de la investigación es contribuir a mejorar la actuación del profesor en su tarea de educar en valores, para ello el trabajo tiene una doble intención, por un lado, realizar una delineación de la axiología educativa relacionándola con el contexto actual de nuestra educación y, por otro parte, aplicar un test basado en el Trait Meta-Mood Scale TMMS-24 para medir la inteligencia emocional de los futuros docentes a través de tres aspectos, atención, claridad y reparación. La investigación anteriormente detallada tenía como objetivo principal examinar la axiología educativa para mejorar la inteligencia emocional y su importancia en la formación de docentes de la carrera de educación básica en la Universidad Técnica de Ambato.

\section{Metodología}

Diseño: El nivel de estudio de la presente investigación fue explicativo, debido a que se busca indagar los niveles de inteligencia emocional en los estudiantes que se están formando para docentes tomando en cuenta la axiología educativa. Se considera de tipo no experimental, ya que no se efectuó manipulación de variable alguna, solo se aplicó el teste validado y se analizó los resultados obtenidos.

Muestra: La observación se ha realizado con una muestra de 106 estudiantes de cuatro niveles de la carrera de Educación Básica de la Universidad Técnica de Ambato Las edades de los participantes oscilaron entre 18 y 23 años. La distribución de estudiantes por curso académico fue: $24.7 \%$ en primero, $27.3 \%$ en segundo, $\%$ en tercero, $\%$ en cuarto, $\%$ en quinto, $\%$ en sexto, $\%$ en séptimo, $\%$ en octavo y $24.3 \%$ en noveno nivel.

Se aplicó un test basado en el Trait Meta-Mood Scale (TMMS-24: Salovey et al., 1995; adaptación al castellano por Fernández-Berrocal, Extremera, y Ramos, 2004), el mismo que consta de 24 preguntas con cinco alternativas que comprenden valores de 1 a 5, así: 1 = Nada de acuerdo; 2 = Algo de acuerdo; 3 = Bastante de acuerdo; 4 = Muy de acuerdo; 5 = Totalmente de acuerdo lo que permitió evaluar la inteligencia emocional de los estudiantes, basándose en tres aspectos, atención, claridad y reparación.

La fiabilidad para cada componente del cuestionario TMMS-24 calculada a través del Alpha de Cronbach, es: Percepción $(\alpha=.90)$; comprensión $(\alpha=.90)$ y regulación $(\alpha=$ 
.86). Asimismo, presenta una fiabilidad test-retest adecuada (Percepción $=.60$; comprensión $=.70$ y regulación $=.83)($ Extremera y Fernández-Berrocal, 2005).

Se procedió analizar la frecuencia de la Inteligencia Emocional observada por los estudiantes calculando las medias y porcentajes de contestación a cada una de las opciones de la escala de Likert en cada ítem. Este análisis se lo efectúo utilizando el programa estadístico SPSS

El test contribuye con un análisis de tres aspectos:

Atención: Hace referencia a la capacidad para examinar nuestras emociones y conocer que significan.

Claridad: Hace énfasis en la potestad para conocer y entender las emociones, integradas a nuestro pensamiento.

Reparación: Hace alusión a la capacidad de normar y reconocer las emociones tanto positivas como negativas.

\section{Resultados}

En la Tabla 1, del análisis efectuado al sexo femenino referente al factor atención se enfatiza una mayor importancia (Muy de acuerdo + Totalmente de acuerdo) a que las estudiantes piensan que merece la pena prestar atención a sus emociones y estado de ánimo $(87,4 \%)$, que presto mucha atención a los sentimientos $(87,3 \%)$ y que normalmente me preocupo mucho por lo que siento (81.0\%). De acuerdo con los intervalos del TMM-24 en la Tabla 2, las estudiantes obtienen un 69,6\% que se traduce en una adecuada atención a sus emociones y el 7,6 presta poca atención a sus emociones.

Tabla 1

Ítems del TMMS-24 en el factor Atención Mujeres

\begin{tabular}{|c|c|c|c|c|c|}
\hline \multirow[b]{2}{*}{ ATENCIÓN MUJERES } & \multicolumn{5}{|c|}{ ESCALA \% } \\
\hline & & & & & \\
\hline 1 & & 2 & 3 & 4 & 5 \\
\hline Presto mucha atención a los sentimientos & $0,0 \%$ & $0,0 \%$ & $12,7 \%$ & $32,9 \%$ & $54,4 \%$ \\
\hline $\begin{array}{l}\text { Normalmente me preocupo mucho por lo que } \\
\text { siento }\end{array}$ & $1,3 \%$ & $2,5 \%$ & $15,2 \%$ & $45,6 \%$ & $35,4 \%$ \\
\hline $\begin{array}{l}\text { Normalmente dedico tiempo a pensar en mis } \\
\text { emociones }\end{array}$ & $1,3 \%$ & $6,3 \%$ & $20,3 \%$ & $39,2 \%$ & $32,9 \%$ \\
\hline $\begin{array}{l}\text { Pienso que merece la pena prestar atención a mis } \\
\text { emociones y estado de ánimo }\end{array}$ & $1,3 \%$ & $3,8 \%$ & $7,6 \%$ & $26,6 \%$ & $60,8 \%$ \\
\hline $\begin{array}{l}\text { Dejo que mis sentimientos afecten a mis } \\
\text { pensamientos }\end{array}$ & $6,3 \%$ & $17,7 \%$ & $29,1 \%$ & $26,6 \%$ & $20,3 \%$ \\
\hline
\end{tabular}


Tabla 1

Ítems del TMMS-24 en el factor Atención Mujeres (continuación)

\begin{tabular}{lrrrrc}
\hline & \multicolumn{5}{c}{ ESCALA \% } \\
\cline { 2 - 6 } ATENCIÓN MUJERES & \multicolumn{1}{c}{1} & \multicolumn{1}{c}{3} & \multicolumn{1}{c}{4} & 5 \\
\hline Pienso en mi estado de ánimo constantemente & $5,1 \%$ & $10,1 \%$ & $26,6 \%$ & $38,0 \%$ & $20,3 \%$ \\
A menudo pienso en mis sentimientos & $3,8 \%$ & $7,6 \%$ & $25,3 \%$ & $36,7 \%$ & $26,6 \%$ \\
Presto mucha atención a cómo me siento & $1,3 \%$ & $3,8 \%$ & $17,7 \%$ & $41,8 \%$ & $35,4 \%$ \\
\hline
\end{tabular}

Nota: Valores de la escala: 1 = Nada de acuerdo; 2 = Algo de acuerdo; 3 = Bastante de acuerdo; 4 = Muy de acuerdo; 5 = Totalmente de acuerdo

Fuente: Encuesta aplicada a los estudiantes de la Carrera de Educación Básica (2021).

\section{Tabla 2}

Ítems del TMMS-24 en el resumen del factor Atención Mujeres

\begin{tabular}{cccccc} 
& Frecuencia & Porcentaje & $\begin{array}{c}\text { Porcentaje } \\
\text { válido }\end{array}$ & $\begin{array}{c}\text { Porcentaje } \\
\text { acumulado }\end{array}$ \\
\cline { 2 - 6 } Válido & Presta poca atención & 6 & 7,6 & 7,6 & 7,6 \\
& $\begin{array}{c}\text { Adecuada atención } \\
\text { Presta demasiada }\end{array}$ & 55 & 69,6 & 69,6 & 77,2 \\
atención & 18 & 22,8 & 22,8 & 100,0 \\
\hline Total & 79 & 100,0 & 100,0 &
\end{tabular}

Fuente: Encuesta aplicada a los estudiantes de la Carrera de Educación Básica (2021).

En la tabla 3, del análisis realizado al sexo masculino referente al factor atención se enfatiza una mayor importancia (Muy de acuerdo + Totalmente de acuerdo) a que las estudiantes piensan que merece la pena prestar atención a sus emociones y estado de ánimo $(88,9 \%)$, que presto mucha atención a los sentimientos $(81,5 \%)$ y que normalmente me preocupo mucho por lo que siento (74.0\%). De acuerdo con los intervalos del TMM-24 en la Tabla 4, los estudiantes obtienen un 55.6\% que se traduce en una adecuada atención a sus emociones y el 3,7 presta poca atención a sus emociones.

Tabla 3

Ítems del TMMS-24 en el factor Atención Hombres

\begin{tabular}{lccccc}
\hline & \multicolumn{5}{c}{ ESCALA \% } \\
\hline ATENCIÓN HOMBRES & 1 & 2 & 3 & 4 & 5 \\
\hline $\begin{array}{l}\text { Presto mucha atención a los sentimientos } \\
\begin{array}{l}\text { Normalmente me preocupo mucho por lo que } \\
\text { siento }\end{array}\end{array}$ & $0,0 \%$ & $3,7 \%$ & $14,8 \%$ & $29,6 \%$ & $51,9 \%$ \\
$\begin{array}{l}\text { Normalmente dedico tiempo a pensar en mis } \\
\text { emociones }\end{array}$ & $0,0 \%$ & $7,4 \%$ & $22,2 \%$ & $33,3 \%$ & $37,0 \%$ \\
$\begin{array}{l}\text { Pienso que merece la pena prestar atención a } \\
\text { mis emociones y estado de ánimo }\end{array}$ & $0,0 \%$ & $3,7 \%$ & $7,4 \%$ & $18,5 \%$ & $70,4 \%$ \\
\hline
\end{tabular}




\section{Tabla 3}

Ítems del TMMS-24 en el factor Atención Hombres (continuación)

\begin{tabular}{lccccc}
\hline \multicolumn{1}{c}{ ATENCIÓN HOMBRES } & 1 & 2 & 3 & 4 & 5 \\
\hline Dejo que mis sentimientos afecten a mis & $0,0 \%$ & $40,7 \%$ & $22,2 \%$ & $22,2 \%$ & $14,8 \%$ \\
pensamientos & $0,0 \%$ & $14,8 \%$ & $25,9 \%$ & $37,0 \%$ & $22,2 \%$ \\
Pienso en mi estado de ánimo constantemente & $0,0 \%$ & $11,1 \%$ & $29,6 \%$ & $33,3 \%$ & $25,9 \%$ \\
A menudo pienso en mis sentimientos & $0,0 \%$ & $7,4 \%$ & $33,3 \%$ & $22,2 \%$ & $37,0 \%$ \\
Presto mucha atención a cómo me siento &
\end{tabular}

Nota: Valores de la escala: 1 = Nada de acuerdo; 2 = Algo de acuerdo; 3 = Bastante de acuerdo; 4 = Muy de acuerdo; 5 = Totalmente de acuerdo

Fuente: Encuesta aplicada a los estudiantes de la Carrera de Educación Básica (2021).

Tabla 4

Ítems del TMMS-24 en el factor Atención Hombres

\begin{tabular}{cccccc}
\hline & & Frecuencia & Porcentaje & $\begin{array}{c}\text { Porcentaje } \\
\text { válido }\end{array}$ & $\begin{array}{c}\text { Porcentaje } \\
\text { acumulado }\end{array}$ \\
\hline \multirow{2}{*}{ Válido } & Presta poca atención & 1 & 3,7 & 3,7 & 3,7 \\
& $\begin{array}{c}\text { Adecuada atención } \\
\text { Presta demasiada }\end{array}$ & 15 & 55,6 & 55,6 & 59,3 \\
& 11 & 40,7 & 40,7 & 100,0 \\
& atención & & & &
\end{tabular}

Fuente: Encuesta aplicada a los estudiantes de la Carrera de Educación Básica (2021).

De la misma forma en la tabla 5, en el factor Claridad emocional referente al sexo femenino, se demuestra valores significativos (Muy de acuerdo y Totalmente de acuerdo) en amenudo me doy cuenta de mis sentimientos en diferentes situaciones $(79,7 \%)$, tengo claro mis sentimientos $(77,2 \%)$ y normalmente conozco mis sentimientos sobre las personas (74,6\%). De acuerdo con los intervalos del TMM-24 en la Tabla 6, las estudiantes obtienen un $57,5 \%$ que se traduce en una adecuada comprensión a sus emociones y el 11,3\% debe mejorar su comprensión. 


\section{Tabla 5}

Ítems del TMMS-24 en el factor Claridad Mujeres

\begin{tabular}{lccccc}
\hline & & \multicolumn{3}{c}{ ESCALA \% } \\
\hline CLARIDAD MUJERES & 1 & 2 & 3 & 4 & 5 \\
\hline Tengo claros mis sentimientos & $2,5 \%$ & $3,8 \%$ & $16,5 \%$ & $30,4 \%$ & $46,8 \%$ \\
Frecuentemente puedo definir mis sentimientos & $3,8 \%$ & $5,1 \%$ & $21,5 \%$ & $40,5 \%$ & $29,1 \%$ \\
Casi siempre sé cómo me siento. & $2,5 \%$ & $2,5 \%$ & $22,8 \%$ & $39,2 \%$ & $32,9 \%$ \\
Normalmente conozco mis sentimientos sobre las & $0,0 \%$ & $5,1 \%$ & $20,3 \%$ & $46,8 \%$ & $27,8 \%$ \\
personas & & & & & \\
A menudo me doy cuenta de mis sentimientos en & $0,0 \%$ & $5,1 \%$ & $15,2 \%$ & $50,6 \%$ & $29,1 \%$ \\
diferentes situaciones & $0,0 \%$ & $12,7 \%$ & $31,6 \%$ & $32,9 \%$ & $22,8 \%$ \\
Siempre puedo decir cómo me siento & $1,3 \%$ & $5,1 \%$ & $29,1 \%$ & $35,4 \%$ & $29,1 \%$ \\
A veces puedo decir cuáles son mis emociones & $0,0 \%$ & $6,3 \%$ & $20,3 \%$ & $41,8 \%$ & $31,6 \%$ \\
Puedo llegar a comprender mis sentimientos &
\end{tabular}

Nota: Valores de la escala: 1 = Nada de acuerdo; 2 = Algo de acuerdo; 3 = Bastante de acuerdo; 4 = Muy de acuerdo; $5=$ Totalmente de acuerdo

Fuente: Encuesta aplicada a los estudiantes de la Carrera de Educación Básica (2021).

\section{Tabla 6}

Ítems del TMMS-24 en el resumen del factor Claridad Mujeres

\begin{tabular}{|c|c|c|c|c|c|}
\hline & & Frecuencia & Porcentaje & $\begin{array}{c}\text { Porcentaje } \\
\text { válido }\end{array}$ & $\begin{array}{l}\text { Porcentaje } \\
\text { acumulado }\end{array}$ \\
\hline \multirow[t]{4}{*}{ Válido } & $\begin{array}{l}\text { Debe mejorar su } \\
\text { comprensión }\end{array}$ & 12 & 11,2 & 11,3 & 11,3 \\
\hline & Adecuada comprensión & 61 & 57,0 & 57,5 & 68,9 \\
\hline & Excelente comprensión & 33 & 30,8 & 31,1 & 100,0 \\
\hline & Total & 106 & 99,1 & 100,0 & \\
\hline \multirow[t]{2}{*}{ Perdidos } & Sistema & 1 & ,9 & & \\
\hline & Total & 107 & 100,0 & & \\
\hline
\end{tabular}

Fuente: Encuesta aplicada a los estudiantes de la Carrera de Educación Básica (2021).

En cuanto a la tabla 7, sobe el mismo factor Claridad emocional, pero en lo que se refiere al sexo masculino, se demuestra valores significativos (Muy de acuerdo y Totalmente de acuerdo) en normalmente conozco mis sentimientos sobre las personas $(74,1 \%)$, a menudo me doy cuenta de mis sentimientos en diferentes situaciones $(74,0 \%)$ y los ítems, tengo claros mis sentimientos como puedo llegar a comprender mis sentimientos coinciden en el porcentaje (70,3\%). De acuerdo con los intervalos del TMM-24 en la Tabla 8 , los estudiantes obtienen un $66,7 \%$ que se traduce en una adecuada comprensión a sus emociones y el $14,8 \%$ debe mejorar su comprensión 


\section{Tabla 7}

Ítems del TMMS-24 en el factor Claridad Hombres

\begin{tabular}{lrrrcc}
\hline & \multicolumn{5}{c}{ ESCALA \% } \\
\hline CLARIDAD HOMBRES & \multicolumn{1}{c}{1} & \multicolumn{1}{c}{3} & 3 & 4 & \multicolumn{1}{c}{5} \\
\hline Tengo claros mis sentimientos & $0,0 \%$ & $7,4 \%$ & $22,2 \%$ & $40,7 \%$ & $29,6 \%$ \\
Frecuentemente puedo definir mis sentimientos & $0,0 \%$ & $3,7 \%$ & $29,6 \%$ & $44,4 \%$ & $22,2 \%$ \\
Casi siempre sé cómo me siento. & $0,0 \%$ & $3,7 \%$ & $18,5 \%$ & $40,7 \%$ & $37,0 \%$ \\
Normalmente conozco mis sentimientos sobre las & $0,0 \%$ & $7,4 \%$ & $18,5 \%$ & $51,9 \%$ & $22,2 \%$ \\
personas & & & & & \\
A menudo me doy cuenta de mis sentimientos en & $0,0 \%$ & $3,7 \%$ & $22,2 \%$ & $44,4 \%$ & $29,6 \%$ \\
diferentes situaciones & $0,0 \%$ & $14,8 \%$ & $25,9 \%$ & $29,6 \%$ & $29,6 \%$ \\
Siempre puedo decir cómo me siento & $0,0 \%$ & $14,8 \%$ & $22,2 \%$ & $29,6 \%$ & $33,3 \%$ \\
A veces puedo decir cuáles son mis emociones & $0,0 \%$ & $7,4 \%$ & $22,2 \%$ & $40,7 \%$ & $29,6 \%$ \\
Puedo llegar a comprender mis sentimientos &
\end{tabular}

Nota: Valores de la escala: 1 = Nada de acuerdo; $2=$ Algo de acuerdo; $3=$ Bastante de acuerdo; 4 = Muy de acuerdo 5 = Totalmente de acuerdo

Fuente: Encuesta aplicada a los estudiantes de la Carrera de Educación Básica (2021).

Tabla 8

Ítems del TMMS-24 en el resumen del factor Claridad Hombres

\begin{tabular}{clcccc}
\hline & Frecuencia & Porcentaje & $\begin{array}{c}\text { Porcentaje } \\
\text { válido }\end{array}$ & $\begin{array}{c}\text { Porcentaje } \\
\text { acumulado }\end{array}$ \\
\hline Válido & $\begin{array}{l}\text { Debe mejorar su } \\
\text { comprensión } \\
\text { Adecuada } \\
\text { comprensión }\end{array}$ & 4 & 14,8 & 14,8 & 14,8 \\
$\begin{array}{l}\text { Excelente } \\
\text { comprensión } \\
\text { Total }\end{array}$ & 5 & 66,7 & 66,7 & 81,5 \\
\hline
\end{tabular}

Fuente: Encuesta aplicada a los estudiantes de la Carrera de Educación Básica (2021).

Para concluir en la Tabla 9, el estudio descriptivo de los ítems que componen el factor reparación emocional se puede apreciar que las estudiantes consideran con mayor medida (Muy de acuerdo + Totalmente de acuerdo) que tiene mucha energía cuando me siento feliz (95\%), que, aunque se sienta mal procura pensar en cosas agradables $(79,7.9 \%)$ y cuando estoy enfadado intento cambiar mi estado de ánimo (78,5\%). En cuanto a los intervalos del TMM-24 en la Tabla 10, las estudiantes obtienen un 49,4\% que se traduce en una adecuada regulación a sus emociones y el 8,9\% debe mejorar su regulación. 


\section{Tabla 9}

Ítems del TMMS-24 en el factor Reparación Mujeres

\begin{tabular}{lllllc}
\hline & & & & ESCALA & \% \\
\hline REPARACIÓN MUJERES & 1 & 2 & 3 & 4 & 5 \\
\hline $\begin{array}{l}\text { Aunque a veces me siento triste, suelo tener una } \\
\text { visión optimista }\end{array}$ & $2,5 \%$ & $8,9 \%$ & $13,9 \%$ & $35,4 \%$ & $39,2 \%$ \\
$\begin{array}{l}\text { Aunque me sienta mal, procuro pensar en cosas } \\
\text { agradables }\end{array}$ & $1,3 \%$ & $5,1 \%$ & $13,9 \%$ & $31,6 \%$ & $48,1 \%$ \\
$\begin{array}{l}\text { Cuando estoy triste, pienso en todos los placeres } \\
\text { de la vida }\end{array}$ & $3,8 \%$ & $10,1 \%$ & $25,3 \%$ & $27,8 \%$ & $32,9 \%$ \\
$\begin{array}{l}\text { Intento tener pensamientos positivos, aunque me } \\
\text { sienta mal. }\end{array}$ & $2,5 \%$ & $5,1 \%$ & $15,2 \%$ & $30,4 \%$ & $46,8 \%$ \\
$\begin{array}{l}\text { Si doy demasiadas vueltas a las cosas, } \\
\text { complicándolas, trato de calmarme }\end{array}$ & $5,1 \%$ & $10,1 \%$ & $19,0 \%$ & $32,9 \%$ & $32,9 \%$ \\
$\begin{array}{l}\text { Me preocupo por tener un buen estado de ánimo } \\
\text { Tengo mucha energía cuando me siento feliz }\end{array}$ & $1,3 \%$ & $5,1 \%$ & $15,2 \%$ & $45,6 \%$ & $32,9 \%$ \\
$\begin{array}{l}\text { Cuando estoy enfadado intento cambiar mi estado } \\
\text { de ánimo. }\end{array}$ & $2,5 \%$ & $5,1 \%$ & $13,9 \%$ & $36,7 \%$ & $41,8 \%$ \\
\hline
\end{tabular}

Nota: Valores de la escala: 1 = Nada de acuerdo; 2 = Algo de acuerdo; 3 = Bastante de acuerdo; 4 = Muy de acuerdo; 5 = Totalmente de acuerdo.

Fuente: Encuesta aplicada a los estudiantes de la Carrera de Educación Básica (2021).

\section{Tabla 10}

Ítems del TMMS-24 en el resumen del factor Reparación Mujeres

\begin{tabular}{llcccc}
\hline & Frecuencia & Porcentaje & $\begin{array}{c}\text { Porcentaje } \\
\text { válido }\end{array}$ & $\begin{array}{c}\text { Porcentaje } \\
\text { acumulado }\end{array}$ \\
\hline \multirow{2}{*}{ Válido } & $\begin{array}{l}\text { Debe mejorar su } \\
\text { regulación }\end{array}$ & 7 & 8,9 & 8,9 & 8,9 \\
& $\begin{array}{l}\text { Adecuada regulación } \\
\text { Excelente regulación }\end{array}$ & 39 & 49,4 & 49,4 & 58,2 \\
& Total & 73 & 41,8 & 41,8 & 100,0 \\
\hline
\end{tabular}

Fuente: Encuesta aplicada a los estudiantes de la Carrera de Educación Básica (2021).

Finalmente, en la Tabla 11, el estudio descriptivo de los ítems que componen el factor reparación emocional referente a los estudiantes del sexo masculino se observa con mayor medida (Muy de acuerdo + Totalmente de acuerdo) que (95\%), que, aunque a veces me siento triste suelo tener una visión optimista al igual que tengo mucha energía cuando me siento feliz coinciden en el porcentaje $(88,9 \%)$ y aunque me sienta mal, procuro pensar en cosas agradables (88,8\%). En cuanto a los intervalos del TMM-24 en la Tabla 12, los estudiantes obtienen un $51,9 \%$ que se traduce en una adecuada regulación a sus emociones y el 7,4 \% debe mejorar su regulación. 


\section{Tabla 11}

Ítems del TMMS-24 en el factor Reparación Hombres

\begin{tabular}{lccccc}
\hline & \multicolumn{5}{c}{ ESCALA \% } \\
\hline REPARACIÓN HOMBRES & 1 & 2 & 3 & 4 & 5 \\
\hline $\begin{array}{l}\text { Aunque a veces me siento triste, suelo tener una } \\
\text { visión optimista }\end{array}$ & $0,0 \%$ & $7,4 \%$ & $3,7 \%$ & $37,0 \%$ & $51,9 \%$ \\
$\begin{array}{l}\text { Aunque me sienta mal, procuro pensar en cosas } \\
\text { agradables }\end{array}$ & $0,0 \%$ & $7,4 \%$ & $3,7 \%$ & $40,7 \%$ & $48,1 \%$ \\
$\begin{array}{l}\text { Cuando estoy triste, pienso en todos los placeres } \\
\text { de la vida }\end{array}$ & $0,0 \%$ & $11,1 \%$ & $22,2 \%$ & $29,6 \%$ & $37,0 \%$ \\
$\begin{array}{l}\text { Intento tener pensamientos positivos, aunque me } \\
\text { sienta mal. }\end{array}$ & $0,0 \%$ & $7,4 \%$ & $0,0 \%$ & $55,6 \%$ & $37,0 \%$ \\
$\begin{array}{l}\text { Si doy demasiadas vueltas a las cosas, } \\
\text { complicándolas, trato de calmarme }\end{array}$ & $0,0 \%$ & $7,4 \%$ & $22,2 \%$ & $40,7 \%$ & $29,6 \%$ \\
$\begin{array}{l}\text { Me preocupo por tener un buen estado de ánimo } \\
\text { Tengo mucha energía cuando me siento feliz }\end{array}$ & $0,0 \%$ & $7,4 \%$ & $22,2 \%$ & $44,4 \%$ & $25,9 \%$ \\
$\begin{array}{l}\text { Cuando estoy enfadado intento cambiar mi } \\
\text { estado de ánimo. }\end{array}$ & $0,0 \%$ & $11,7 \%$ & $7,4 \%$ & $33,3 \%$ & $55,6 \%$ \\
\end{tabular}

Nota: Valores de la escala: 1 = Nada de acuerdo; 2 = Algo de acuerdo; 3 = Bastante de acuerdo; $4=$ Muy de acuerdo; $5=$ Totalmente de acuerdo.

Fuente: Encuesta aplicada a los estudiantes de la Carrera de Educación Básica (2021)

\section{Tabla 12}

Ítems del TMMS-24 en el resumen del factor Reparación Hombres

\begin{tabular}{llcccc}
\hline & Frecuencia & Porcentaje & $\begin{array}{c}\text { Porcentaje } \\
\text { válido }\end{array}$ & $\begin{array}{c}\text { Porcentaje } \\
\text { acumulado }\end{array}$ \\
\hline Válido & Debe mejorar su & 2 & 7,4 & 7,4 & 7,4 \\
& regulación & 14 & 51,9 & 51,9 & 59,3 \\
& $\begin{array}{l}\text { Adecuada regulación } \\
\text { Excelente regulación }\end{array}$ & 11 & 40,7 & 40,7 & 100,0 \\
\hline & Total & 27 & 100,0 & 100,0 &
\end{tabular}

Fuente: Encuesta aplicada a los estudiantes de la Carrera de Educación Básica (2021)

\section{Conclusiones}

- En lo que respecta a la inteligencia emocional los resultados obtenidos determinan que los estudiantes de la carrera de educación básica poseen un apropiado nivel de IE con diferencias entre las tres dimensiones analizadas, destacando una puntuación mayor en la reparación emocional a continuación 
la atención emocional y finalmente la claridad emocional.

- Dentro de la reparación emocional tanto para mujeres y hombres hay que destacar el tema que tiene mucha energía cuando me siento feliz $(95 \%)$. En cuanto a los intervalos del TMM-24 las estudiantes obtienen un 49,4\% que se traduce en una adecuada regulación a sus emociones y el 8,9\% debe mejorar su regulación

- En atención mujeres y hombres presenta mayores valoraciones al manifestar que piensan que merece la pena prestar atención a sus emociones y estado de ánimo. De acuerdo con los intervalos del TMM-24 las estudiantes mujeres obtienen un $69,6 \%$ que se traduce en una adecuada atención a sus emociones y el 7,6 presta poca atención a sus emociones y los estudiantes hombres alcanzan un 55.6\% que se traduce en una adecuada atención a sus emociones y el 3,7 presta poca atención a sus emociones

- Finalmente, en claridad emocional mujeres a menudo me doy cuenta de mis sentimientos en diferentes situaciones y claridad emocional hombres normalmente conozco mis sentimientos sobre las personas registran los mayores porcentajes. De acuerdo con los intervalos del TMM-24 las estudiantes, obtienen un 57,5\% que se traduce en una adecuada comprensión a sus emociones y el 11,3\% debe mejorar su comprensión, en cuanto a los estudiantes registran un $66,7 \%$ que se traduce en una adecuada comprensión a sus emociones y el 14,8\% debe mejorar su comprensión.

- En síntesis, se puede afirmar que existe relación entre la axiología educativa y la inteligencia emocional orientada a la formación de futuros docentes, promoviendo la educación en valores, esto involucra condiciones en los diferentes espacios de educación formal, no formal e informal por medio de las interacciones sociales. En la construcción de valores la escuela y el docente se convierten en instancias importantes en la educación de futuros ciudadanos, sin embargo, no son las únicas fuentes de referencia.

\section{Referencias bibliográficas}

Contreras, P. (2010). "Ser y saber en la formación didáctica del profesorado". Revista Interuniversitaria de Formación del Profesorado, 68 (24,2), 61-81.

Delors, J. (1997). La educación encierra un tesoro: informe para la UNESCO de la Comisión Internacional sobre la Educación para el Siglo Veintiuno. UNESCO.

Dewey, J. (1938). Experience and Education. Collier Books.

Exposito, C. (2018). Valores básicos del profesorado: una aproximación desde el modelo 
axiológico de Shalom Schwortz. Educación y educadores.

Genis, O. (2005). Valores y narrativa: axiología educativa occidente. Edición Universitat Barcelona.

Hernández, P. (2005). Eduacción del pensamiento y de las emociones.

Imbernón, F. (2001), “Claves para una nueva formación del profesorado”, Investigación en la Escuela, núm. 43, pp. 57-66.

Korthagen, F., Loughran, J. \& Russell, T. (2006). "Developing fundamental principles for teacher education programs and practices". Teaching and Teacher Education, 22(8), 1020-1041.

Marin, G. (1987). Crecimiento personal y desarrollo de valores. Un nuevo enfoque educativo. Promolibro.

Mayer, J. (1997). What is emotional inteligence. Emotional development and emotional intelligence.

Piotrowski, B. (1996). La Axiología y la educación. Educación - Educadores, 131.

Salovey, P. (1990). Emotional inteligence. Imagination, Cognition, and Personality. 185 $-211$.

Santomé, J. (1995). Curriculum oculto. 3. ed. Porto Editora.

Tardif, M. (2013). Las políticas de formación docente en América Latina. Avances y Desafios Pendientes. En M. Poggi, Políticas docentes: formación, trabajo y desarrollo profesional (pp. 19-45). Unesco.

Torres, J. (1998) El currículum oculto. "El mundo visto desde las instituciones escolares: La lucha contra la exclusión", en Educación, desarrollo y participación democrática. Proyecto y tú... ¿cómo la ves? ACSUR-Las Segovias, pp. 77-90.

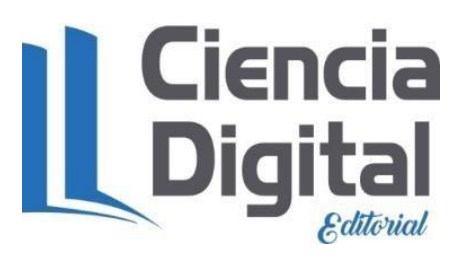


El artículo que se publica es de exclusiva responsabilidad de los autores y no necesariamente reflejan el pensamiento de la Revista Conciencia Digital.

\section{Liencia}

El artículo queda en propiedad de la revista y, por tanto, su publicación parcial y/o total en otro medio tiene que ser autorizado por el director de la Revista Conciencia Digital.
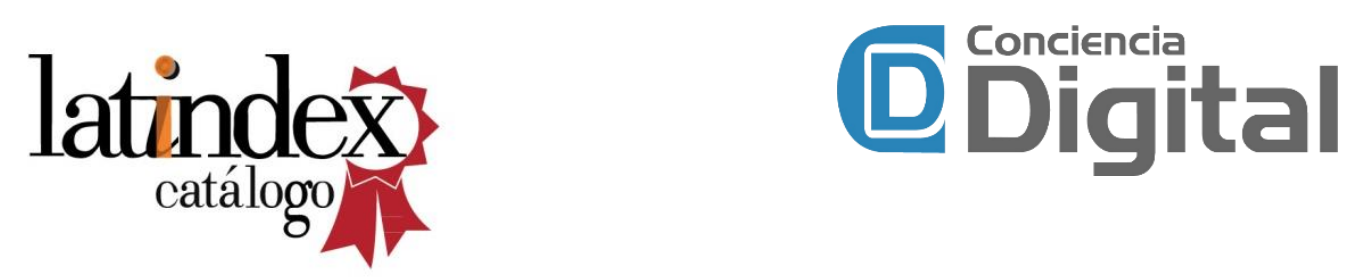

Indexaciones

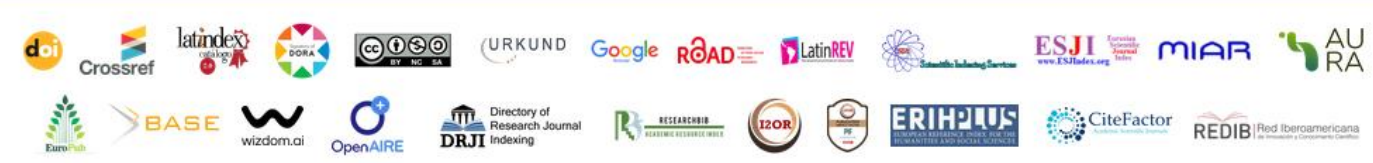

